\title{
ON MORITA'S FUNDAMENTAL THEOREM FOR $\mathrm{C}^{*}$-ALGEBRAS
}

\author{
DAVID P. BLECHER
}

\begin{abstract}
We give a solution, via operator spaces, of an old problem in the Morita equivalence of $\mathrm{C}^{*}$-algebras. Namely, we show that $\mathrm{C}^{*}$-algebras are strongly Morita equivalent in the sense of Rieffel if and only if their categories of left operator modules are isomorphic via completely contractive functors. Moreover, any such functor is completely isometrically isomorphic to the Haagerup tensor product (= interior tensor product) with a strong Morita equivalence bimodule. An operator module over a $\mathrm{C}^{*}$-algebra $\mathscr{A}$ is a closed subspace of some $\mathrm{B}(\mathrm{H})$ which is left invariant under multiplication by $\pi(\mathscr{A})$, where $\pi$ is a *-representation of $\mathscr{A}$ on $H$. The category $\mathscr{A} H M O D$ of *-representations of $\mathscr{A}$ on Hilbert space is a full subcategory of the category $\mathscr{A} O M O D$ of operator modules. Our main result remains true with respect to subcategories of $O M O D$ which contain $H M O D$ and the $\mathrm{C}^{*}$-algebra itself. It does not seem possible to remove the operator space framework; in the very simplest cases there may exist no bounded equivalence functors on categories with bounded module maps as morphisms (as opposed to completely bounded ones). Our proof involves operator space techniques, together with a $\mathrm{C}^{*}$-algebra argument using compactness of the quasistate space of a $\mathrm{C}^{*}$-algebra, and lowersemicontinuity in the enveloping von Neumann algebra.
\end{abstract}

\section{Notation, background and statement of the theorem}

In the early 70's M. Rieffel introduced and developed the notion of strong Morita equivalence of $\mathrm{C}^{*}$-algebras (see [26] for a good discussion and survey). It has become a fundamental tool in modern operator algebra and noncommutative geometry (see [12] for example). Briefly, two $\mathrm{C}^{*}$-algebras $\mathscr{A}$ and $\mathscr{B}$ are said to be strongly Morita equivalent if there is an $\mathscr{A}-\mathscr{B}$-bimodule $X$, which is a right $\mathrm{C}^{*}$-module over $\mathscr{B}$, and a left $\mathrm{C}^{*}$-module over $\mathscr{A}$, such that the inner products $\mathscr{A}\langle\cdot \mid \cdot\rangle$ and $\langle\cdot \mid \cdot\rangle_{\mathscr{B}}$ satisfy the relation $\mathscr{A}\left\langle x_{1} \mid x_{2}\right\rangle x_{3}=x_{1}\left\langle x_{2} \mid x_{3}\right\rangle_{\mathscr{B}}$, for $x_{1}, x_{2}, x_{3} \in X$. Also the span of the range of these inner products must be norm dense in $\mathscr{A}$ and $\mathscr{B}$ respectively. Such $X$ is said to be an $\mathscr{A}-\mathscr{B}$-strong Morita equivalence bimodule.

Our main result is a $\mathrm{C}^{*}$-algebraic version of Morita's fundamental theo-

Received June 6, 1997, in revised form May 4, 1998.

* Supported by a grant from the NSF

The contents of this paper were announced at the joint meeting of the Canadian Operator Algebra Symposium, and the Great Plains Operator Theory Seminar, May 17-22, 1997 
rem from pure algebra. Namely, we show that two $\mathrm{C}^{*}$-algebras are strongly Morita equivalent if and only if their categories of (left) operator modules are isomorphic via completely contractive functors. Moreover, any such functor is completely isometrically isomorphic to the Haagerup tensor product (= interior tensor product) with a strong Morita equivalence bimodule. We use the context of operator spaces. In previous papers [9, 8, 7] we showed that operator spaces, and more particularly operator modules, are an appropriate 'metric' context for the $\mathrm{C}^{*}$-algebraic theory of strong Morita equivalence and the related theory of $\mathrm{C}^{*}$-modules. Thus it was natural to look for a 'fundamental Morita theorem' in this category.

Let us begin by establishing the common symbols and notations in this paper. We shall use operator spaces quite extensively, and their connections to $\mathrm{C}^{*}$-modules. We refer the reader to [8] and [18] for missing background. The algebraic background may be found in any account of Morita theory for rings, such as [1]. We have deliberately supressed some of the purely algebraic calculations, since sentences consisting of long strings of natural isomorphisms are not particularly interesting or enlightening. None of these supressed calculations are difficult, and hopefully can be supplied without too much trouble by the reader.

We will use the symbols $\mathscr{A}, \mathscr{B}$ for $\mathrm{C}^{*}$-algebras; $a, b$ will be generic elements of $\mathscr{A}$ and $\mathscr{B}$ respectively; and $\left\{e_{\alpha}\right\},\left\{f_{\beta}\right\}$ are contractive approximate identities (c.a.i.'s) for $\mathscr{A}$ and $\mathscr{B}$ respectively. We write $e(\mathscr{A})$ for the enveloping von Neumann algebra of $\mathscr{A} . H, K, \cdots$ are Hilbert spaces, $\zeta, \eta$ are typical elements in $H$ and $K$ respectively, and $B(H)$ (resp. $B(H, K)$ ) is the space of bounded linear operators on $H$ (resp. from $H$ to $K$ ). We will reserve the symbols $Y$ and $Z$ for a right $\mathscr{A}$-module, or a left $\mathscr{B}$-module, or an $\mathscr{B}-\mathscr{A}$ bimodule; it has generic element $y$ and $z$ respectively. Similarly, $X$ or $W$ will be a right $\mathscr{B}$-, left $\mathscr{A}$-, or $\mathscr{A}-\mathscr{B}$-module, with generic element $x$ or $w$.

Suppose that $\pi$ is a *-representation of $\mathscr{A}$ on Hilbert space $H$, and that $X$ is a closed subspace of $B(H)$ such that $\pi(\mathscr{A}) X \subset X$. Then $X$ is a left $\mathscr{A}$ module. We shall assume that the module action is nondegenerate (= essential) ${ }^{1}$. We say that such $X$, considered as an abstract operator space and a left $\mathscr{A}$-module, is a left operator module over $\mathscr{A}$. By considering $X$ as an abstract operator space and module, we may forget about the particular $H, \pi$ used $^{2}$. A theorem of Christensen-Effros-Sinclair [13] tells us that the operator modules are exactly the operator spaces which are (nondegenerate)

\footnotetext{
${ }^{1}$ This means (for a left Banach module $X$ over $\mathrm{A}$, say) that $\left\{\sum_{k=1}^{n} a_{k} x_{k}: n \in \mathrm{N}\right.$, $\left.a_{k} \in \mathrm{A}, x_{k} \in X\right\}$ is dense in $X$. This is equivalent to saying that for any c.a.i. $\left\{e_{\alpha}\right\}$ in $\mathrm{A}, e_{\alpha} x \rightarrow x$ for all $x \in X$.

${ }^{2}$ It is sometimes useful, and equivalent, to allow $X$ in the definition above, to be a subspace of $B(K, H)$, for a second Hilbert space $K$.
} 
left $\mathscr{A}$-modules, such that the module action is a 'completely contractive' bilinear map (or equivalently, the module action linearizes to a complete contraction $\mathscr{A} \otimes_{h} X \rightarrow X$, where $\otimes_{h}$ is the Haagerup tensor product). We will use the facts that submodules and quotient modules of operator modules, are again operator modules. Also, if $X$ is a left operator module and $E$ is an operator space, then the Haagerup tensor product $X \otimes_{h} E$ is a left operator module. This last fact follows easily from the last definition of an operator module in terms of the Haagerup tensor product, and the fact that that tensor product is associative. We write $\mathscr{A} O M O D$ for the category of left $\mathscr{A}$-operator modules. The morphisms are ${ }_{\mathscr{A}} \mathrm{CB}(X, W)$, the completely bounded left $\mathscr{A}$-module maps.

We now turn to the category $\mathscr{A} H M O D$ of Hilbert spaces $H$ which are left $\mathscr{A}$-modules via a nondegenerate $*$-representation of $\mathscr{A}$ on $H$ (denoted $\mathscr{A}$ Hermod in [24]). If $H$ is a Hilbert space, and if $e_{0}$ is a fixed unit vector in $H$, then the space of rank 1 operators $H^{c}=\left\{\zeta \otimes e_{0} \in B(H): \zeta \in H\right\}$ is clearly an operator space, and indeed is clearly in $\mathscr{A} O M O D$ if $H \in \mathscr{A} H M O D$. As an operator space or operator module $H^{c}$ is independent of the particular $e_{0}$ we picked. It is referred to in the literature as 'Hilbert column space'. The $n$-dimensional Hilbert column space is written as $C_{n}$. It is well known that for a linear map $T: H \rightarrow K$ between Hilbert spaces, the usual norm equals the completely bounded norm of $T$ as a map $H^{c} \rightarrow K^{c}$. Thus we see that the assignment $H \mapsto H^{c}$ embeds $\mathscr{A} H M O D$ as a subcategory of $\mathscr{A} O M O D$. Henceforth we will view it as a subcategory.

It is explained in [27] that $\mathrm{C}^{*}$-modules also possess canonical operator space structures, and so can be viewed as objects in $O M O D$. In [8] this idea is developed and, amongst other things, we showed that the well known interior tensor product of $\mathrm{C}^{*}$-modules coincides with their Haagerup tensor product as operator modules. This fact is important in what follows.

If $X, W \in \mathscr{A} O M O D$ then $\mathscr{A} \mathrm{CB}(X, W)$ is an operator space with $M_{n}\left({ }_{\mathscr{A}} \mathrm{CB}(X, W)\right) \cong{ }_{\mathscr{A}} \mathrm{CB}\left(X, M_{n}(W)\right)$ [14]. In this paper we are concerned with functors between categories of operator modules. Such functors $F$ : $\mathscr{A} O M O D \rightarrow \mathscr{B} O M O D$ are assumed to be linear on spaces of morphisms. Thus $T \mapsto F(T)$ from ${ }_{\mathscr{A}} \mathrm{CB}(X, W) \rightarrow{ }_{\mathscr{B}} \mathrm{CB}(F(X), F(W))$ is linear, for all pairs of objects $X, W \in{ }_{A} O M O D$. We say $F$ is completely contractive, if this map $T \mapsto F(T)$ is completely contractive, for all pairs of objects $X, W \in$ $\mathscr{A} O M O D$. We say two functors $F_{1}, F_{2}: \mathscr{A} O M O D \rightarrow_{\mathscr{B}} O M O D$ are (naturally) completely isometrically isomorphic, if they are naturally isomorphic in the sense of category theory [1], with the natural transformations being complete isometries. In this case we write $F_{1} \cong F_{2}$ completely isometrically.

Definition 1.1. We say that two $\mathrm{C}^{*}$-algebras $\mathscr{A}$ and $\mathscr{B}$ are operator 
Morita equivalent if there exist completely contractive functors $F$ : $\mathscr{A} O M O D \rightarrow{ }_{\mathscr{B}} O M O D$ and $G: \mathscr{B} O M O D \rightarrow \mathscr{A} O M O D$, such that $F G \cong \mathrm{Id}$ and $G F \cong$ Id completely isometrically. Such $F$ and $G$ will be called operator equivalence functors.

We can now state our main theorem. Its proof, which occupies $\S 2$ and 3, involves operator space techniques, together with a $\mathrm{C}^{*}$-algebra argument using compactness of the quasistate space $Q$ of a $\mathrm{C}^{*}$-algebra, and lowersemicontinuity in the enveloping von Neumann algebra.

THeORem 1.2. Two $C^{*}$-algebras $\mathscr{A}$ and $\mathscr{B}$ are strongly Morita equivalent if and only if they are operator Morita equivalent. Suppose that $F, G$ are the operator equivalence functors, and set $Y=F(\mathscr{A})$ and $X=G(\mathscr{B})$. Then $X$ is an $\mathscr{A}-\mathscr{B}$-strong Morita equivalence bimodule, $Y$ is a $\mathscr{B}-\mathscr{A}$-strong Morita equivalence bimodules, and $Y$ is unitarily equivalent to the conjugate $C^{*}$-bimodule $\bar{X}$ of $X$. Moreover, $F(W) \cong Y \otimes_{h \mathscr{A}} W \cong \mathscr{A} \mathrm{K}(X, W)$ completely isometrically isomorphically (as $\mathscr{B}$-operator modules), for all $W \in \mathscr{A} O M O D$. Thus $F \cong Y \otimes_{h \mathscr{A}}-\cong{ }_{\mathscr{A}} \mathrm{K}(X,-)$ completely isometrically. Similarly $G \cong X \otimes_{h \mathscr{B}}-\cong \mathscr{B} \mathrm{K}(Y,-)$ completely isometrically. Also $F$ maps the subcategory ${ }_{\mathscr{A}} H M O D$ to $\mathscr{B} H M O D$, and the subcategory of $C^{*}$-modules to the $C^{*}$ modules (on which subcategories the Haagerup tensor product above coincides with the interior tensor product). Similar statements hold for $G$.

We remind the reader that $\mathscr{A} \mathrm{K}(X, W)$ was defined in [8] to be the norm closure in $\mathscr{A} \mathrm{CB}(X, W)$ of the span of the rank one operators $\langle\cdot \mid x\rangle w$, for $x \in X, w \in W$. The symbol $\otimes_{h, d}$ denotes the module Haagerup tensor product over $\mathscr{A}$ (see [9] or [8]).

REMARK 1 . The one direction of the "if and only if" of the theorem is easy and was noted in [9]. For completeness we sketch the short argument here. Namely, if $X$ is a strong Morita equivalence bimodule for a strong Morita equivalence of $\mathscr{A}$ and $\mathscr{B}$, and if $Y=\bar{X}$ is the conjugate $\mathrm{C}^{*}$-module, then define $F(W)=Y \otimes_{h \mathscr{A}} W$, and $G(Z)=X \otimes_{h \mathscr{B}} Z$. Since the Haagerup tensor product is functorial, $F$ and $G$ are functors. By the associativity of the module Haagerup tensor product, and the fact that this tensor product equals the interior tensor product where the latter is defined, we obtain that

$$
G F(W) \cong X \otimes_{h \mathscr{B}}\left(Y \otimes_{h \mathscr{A}} W\right) \cong\left(X \otimes_{h \mathscr{B}} Y\right) \otimes_{h \mathscr{A}} W \cong \mathscr{A} \otimes_{h \mathscr{A}} W \cong W
$$

completely isometrically, and as $\mathscr{A}$-modules. Similarly $F G \cong I d$ completely isometrically. So $\mathscr{A}$ and $\mathscr{B}$ are operator Morita equivalent.

Remark 2. One can adapt the statement of the theorem to allow the operator equivalence functors to be defined on not all of $O M O D$, but only on a subcategory $\mathbf{D}$ of $O M O D$ which contains $H M O D$ and the $\mathrm{C}^{*}$-algebra itself. 
Our proof goes through verbatim, except that for the part in $\S 2$ that equivalence functors preserve $H M O D$. For this part to work, the subcategory $\mathbf{D}$ should be closed under two or three operations which we leave to the interested reader to abstract.

We also remark that the proof would become a little simpler if we are willing to assume that the functors concerned are 'strongly continuous' (by which we mean that $F\left(T_{\lambda}\right)$ converges point norm to $F(T)$ whenever $T_{\lambda}$ is a bounded net in ${ }_{\mathscr{A}} C B(X, W)$ converging point norm to $\left.T\right)$. This argument, which was in the original version of this paper, has been omitted for the sake of brevity.

REMARK 3. The reader may question the necessity of using operator spaces, and completely contractive or completely isometric maps and functors. However it is not too hard to show that even in the very simplest case, where $\mathscr{A}=\mathrm{C}, \mathscr{B}=M_{n}$ (the $n \times n$ scalar matrices), and if we write $\mathbf{D}$ for either the category of left Banach modules, or the category of operator modules but with bounded module maps as opposed to completely bounded ones, that there exists no isometric equivalence functor $F: \mathscr{A} \mathbf{D} \rightarrow \mathscr{B} \mathbf{D}$. In these categories there are too many morphisms; one needs to restrict attention to the completely bounded ones. If one replaces $\mathscr{B}$ by the compact operators on $\ell^{2}$, there exists no bounded equivalence functor (see also [17]).

Indeed one runs into problems using bounded module maps as morphisms if one picks the smallest categories containing $H M O D$ and the algebra itself. Namely, suppose that $\mathscr{A}$ and $\mathscr{B}$ are strongly Morita equivalent, with $\mathscr{A}-\mathscr{B}$ equivalence bimodule $X$ and dual bimodule $Y \cong \bar{X}$. Let ${ }_{\mathscr{A}} \mathbf{C}$ be the category of left Banach (or operator) $\mathscr{A}$-modules consisting of $\mathscr{A} H M O D, \mathscr{A}$ and $X$ (the latter two viewed as left $\mathscr{A}$-modules). Let $\mathscr{B} \mathbf{D}$ consist of $\mathscr{B} H M O D, B$ and $Y$. Morphisms in both categories are the bounded module maps. Take $F$ to be the obvious functor, namely the one that maps $\mathscr{A}$ to $Y, X$ to $\mathscr{B}$, and on $\mathscr{A} H M O D$ is the interior tensor product with $Y$. Define $G: \mathscr{B} \mathbf{D} \rightarrow \mathscr{A} \mathbf{C}$ similarly. Again it is easy to check that even in the simplest cases $F$ and $G$ are not necessarily contractive or bounded.

Remark 4. W. Beer proved in [5] that two unital $\mathrm{C}^{*}$-algebras are strongly Morita equivalent if and only if they are algebraically Morita equivalent. Our theorem may be viewed as an extension to the general case which also has the advantage of characterizing the equivalence functors up to (complete) isometry. Also, in Beer's theorem one produces the $\mathrm{C}^{*}$-module by finding a similarity of an idempotent in a matrix algebra to a selfadjoint idempotent, whereas our equivalence bimodule comes directly from the functor.

In [8] we gave another $\mathrm{C}^{*}$-algebraic analogue of Morita's fundamental 
theorem in terms of categories of $\mathrm{C}^{*}$-modules; but that theorem was much less satisfying. The definition of a $\mathrm{C}^{*}$-module is not too far from that of a strong Morita equivalence, so that while that theorem was not quite tautological, it was certainly not very deep ${ }^{3}$. It seems much more surprizing, at least to us, that strong Morita equivalence should be related to the category of operator modules. After all, the notion of an operator module has nothing to do with the notion of strong Morita equivalence. Another 'drawback' of the theorem in [8] is that the category of $\mathrm{C}^{*}$-modules does not contain the category $\mathscr{A} H M O D$ of Hilbert space modules.

\section{Preliminary Lemmas}

Throughout this section $\mathscr{A}$ and $\mathscr{B}$ are $\mathrm{C}^{*}$-algebras, and $F: \mathscr{A} O M O D \rightarrow$ ${ }_{\mathscr{B}} O M O D$ is an operator equivalence functor, with 'inverse' $G$ (see Definition 1.1). We set $Y=F(\mathscr{A})$ and $X=G(\mathscr{B})$. For a a left module $W$ over $\mathscr{A}$, say, and $w \in W$, we write $r_{w}$ for the map from $\mathscr{A} \rightarrow W$ which is simply right multiplication by $w$.

Lemma 2.1. Let $W \in \mathscr{A} O M O D$. Then $w \mapsto r_{w}$ is a complete isometry of $W$ into $\mathscr{A} \mathrm{CB}(\mathscr{A}, W)$. Indeed, $W$ is completely isometrically isomorphic to $\left\{T \in \mathscr{A} \mathrm{CB}(\mathscr{A}, W): T r_{e_{\alpha}} \rightarrow T\right.$ in norm $\}$, where $\left\{e_{\alpha}\right\}$ is a c.a.i. for $\mathscr{A}$. If $W$ is also a Hilbert space, then the map above is a completely isometric isomorphism $W \cong \mathscr{A} \mathrm{CB}(\mathscr{A}, W)$.

This is a simple consequence of the existence of a c.a.i. in any $\mathrm{C}^{*}$-algebra see [28]. The following lemma will be used extensively without comment. It's proof is just as in pure algebra ([1] Proposition 21.2).

LEMma 2.2. If $V, W \in \mathscr{A} O M O D$ then the map $T \mapsto F(T)$ gives a completely isometric surjective linear isomorphism ${ }_{\mathscr{A}} \mathrm{CB}(V, W) \cong{ }_{\mathscr{B}} C B(F(V), F(W))$. If $V=W$ this map is a completely isometric isomorphism of algebras.

If $E$ is an operator space, then the space $M_{m, n}(E)$ of $m \times n$ matrices with entries in $E$, is also an operator space in a canonical way. We write $C_{m}(E)$ and $R_{m}(E)$ for the operator spaces $M_{m, 1}(E)$ and $M_{1, m}(E)$. If $W \in \mathscr{A} O M O D$, then it is easy to see that $R_{m}(W)$ and $C_{m}(W)$ are again in $\mathscr{A} O M O D$.

For $n=1, \cdots, m$, write $i_{n}$ (resp. $\pi_{n}$ ) for the canonical coordinatewise inclusion (resp. projection) map of $W$ into the direct sum $C_{m}(W)$ or $R_{m}(W)$ (resp. from the direct sum onto $W$ ). Then $\pi_{n} i_{k}=\delta_{k, n} I \mathrm{~d}_{W}$ for each $n, k$ (where $\delta_{k, n}$ is the Kronecker delta), and $\sum_{n} i_{n} \pi_{n}=$ Id. Applying the functor $F$ gives maps $F\left(i_{n}\right): F(W) \rightarrow F\left(R_{m}(W)\right)$, and $F\left(\pi_{n}\right): F\left(R_{m}(W)\right) \rightarrow F(W)$, with

\footnotetext{
${ }^{3}$ Indeed the proof of the aforementioned theorem in [8] is rather too long: as we noted in the galley proofs to that paper, G. Skandalis has shown us a shorter proof.
} 
$F\left(\pi_{n}\right) F\left(i_{k}\right)=\delta_{k, n} \mathrm{Id}$ for each $n, k$, and $\sum_{n} F\left(i_{n}\right) F\left(\pi_{n}\right)=$ Id. These formulae yield a canonical algebraic isomorphism $F\left(R_{m}(W)\right) \cong R_{m}(F(W))$. Similarly in the $C_{m}(W)$ case. We now prove these isomorphisms are completely isometric:

Lemma 2.3. For any $W \in \mathscr{A}_{O} O M O D$, we have $F\left(R_{m}(W)\right) \cong R_{m}(F(W))$ and $F\left(C_{m}(W)\right) \cong C_{m}(F(W))$ completely isometrically isomorphically.

Proof. In the $R_{m}(W)$ case, note $\left[\pi_{1}, \cdots, \pi_{m}\right] \in R_{m}\left({ }_{\mathscr{A}} \mathrm{CB}\left(R_{m}(W), W\right)\right)$, and it has norm 1 (as may be seen by noting that it corresponds to the identity map after employing the canonical completely isometric identification $\left.R_{m}\left({ }_{\mathscr{A}} \mathrm{CB}\left(R_{m}(W), W\right)\right) \cong{ }_{\mathscr{A}} \mathrm{CB}\left(R_{m}(W), R_{m}(W)\right)\right)$. Applying $F$, we find $J=\left[F\left(\pi_{1}\right), \cdots, F\left(\pi_{m}\right)\right] \in R_{m}\left({ }_{\mathscr{B}} C B\left(F\left(R_{m}(W)\right), F(W)\right)\right)$ has norm 1. However, via the canonical completely isometric isomorphism of $R_{m}\left({ }_{\mathscr{B}} \mathrm{CB}\left(F\left(R_{m}(W)\right), F(W)\right)\right)$ with $\mathscr{B}_{\mathscr{B}} \mathrm{CB}\left(F\left(R_{m}(W)\right), R_{m}(F(W))\right), \quad J$ corresponds to the canonical morphism $F\left(R_{m}(W)\right) \rightarrow R_{m}(F(W))$. So this latter morphism is a complete contraction. Similarly the canonical morphism $G\left(R_{m}(F(W))\right) \rightarrow R_{m}(G F(W)) \cong R_{m}(W)$ is a complete contraction. Applying $F$ to this morphism, gives a complete contraction $F G\left(R_{m}(F(W))\right) \rightarrow$ $F\left(R_{m}(W)\right)$, which yields a complete contraction $R_{m}(F(W)) \rightarrow F\left(R_{m}(W)\right)$. This proves the lemma for $R_{m}(W)$. The $C_{m}(W)$ case is similar.

In the remainder of this section we show that $F$ takes the subcategory $\mathscr{A} H M O D$ to $\mathscr{B} H M O D$, and similarly for $G$. Choose $H \in \mathscr{A} H M O D$, and recall that $H$ may be identified with $H^{c} \in \mathscr{A} O M O D$. We will show that $F\left(H^{c}\right) \in{ }_{\mathscr{B}} H M O D$, or equivalently, that $F\left(H^{c}\right)$ is a column Hilbert space. For this we need the following functorial characterization of column Hilbert space:

Proposition 2.4. Let E be an operator space. Then $E$ is completely isometrically isomorphic to a Hilbert column space if and only if the identity map $E \otimes_{\min } C_{m} \rightarrow E \otimes_{h} C_{m}$ is a complete contraction for all $m \in \mathrm{N}$.

Proof. The $(\Rightarrow)$ direction is easy and is omitted $[6,11,15]$. A simple proof of the other direction may be found in [16] (Theorem 4.1, setting $q=1$ ). For completeness, we sketch a slight simplification of their argument. We use canonical operator space identifications, which may be found in $[11,15,6]$, and the notation of [6]. By the complete injectivity of the minimal and Haagerup tensor product, (see [11] for example), and the fact that column Hilbert space is determined by its finite dimensional subspaces being column space, it follows that $E \otimes_{\min } H^{c}=E \otimes_{h} H^{c}$, for any Hilbert space $H$. Choose $H$ so that $E \subset B(H)$. The last "=" may be rewritten as $H^{c} \otimes_{h} E=H^{c} \widehat{\otimes}$, where $\widehat{\otimes}$ is the operator space projective tensor product. Next, recall that the 
functors $H^{r} \otimes_{h}-$ and $H^{r} \otimes-$ are the same. Applying this functor to the identity $H^{c} \otimes_{h} E=H^{c} \otimes E$ yields the identity $S_{1}(H) \otimes_{h} E=S_{1}(H) \otimes E$, where $S_{1}(H)$ is the operator space predual of $B(H)$. Taking the operator space dual yields $\mathrm{CB}(E, B(H))=\Gamma^{c}(E, B(H))$. Thus the inclusion map $E \subset B(H)$ factors through Hilbert column space. Hence $E$ is Hilbert column space.

We remark that Pisier has shown us that the last result is true with the word "complete" removed.

To use this to prove that $K=F\left(H^{c}\right)$ is a column Hilbert space, we first remind the reader that for Hilbert column spaces, all operator space tensor norms coincide [15,6], thus $C_{m}\left(H^{c}\right) \cong H^{c} \otimes_{\min } C_{m} \cong H^{c} \otimes_{h} C_{m}$ completely isometrically. Using this and Lemma 2.3 we have (completely isometrically):

$(*) \quad K \otimes_{\min } C_{m} \cong C_{m}\left(F\left(H^{c}\right)\right) \cong F\left(C_{m}\left(H^{c}\right)\right) \cong F\left(H^{c} \otimes_{h} C_{m}\right) \cong F\left(G(K) \otimes_{h} C_{m}\right)$

since $G(K) \cong H^{c}$. Next we remark that there is a canonical complete contraction $G(K) \otimes_{h} C_{m} \rightarrow G\left(K \otimes_{h} C_{m}\right)$. To explain this map, first consider the map $G(K) \rightarrow{ }_{\mathscr{B}} \mathrm{CB}(Y, K)$ given by the following sequence of maps:

$$
G(K) \rightarrow \mathscr{A} \mathrm{CB}(\mathscr{A}, G(K)) \cong{ }_{\mathscr{B}} \mathrm{CB}(Y, F G(K)) \cong{ }_{\mathscr{B}} \mathrm{CB}(Y, K) .
$$

The $\rightarrow$ in $(* *)$ comes from Lemma 2.1 , whereas the $\cong$ comes from applying the equivalence functor (see Lemma 2.2). Using $(* *)$ we get a sequence of completely contractive module maps:

$$
\begin{aligned}
(* * *) \quad G(K) \otimes_{h} C_{m} & \rightarrow{ }_{\mathscr{B}} \mathrm{CB}(Y, K) \otimes_{h} C_{m} \rightarrow \mathscr{B} \mathrm{CB}\left(Y, K \otimes_{h} C_{m}\right) \\
& \cong \mathscr{A} \mathrm{CB}\left(\mathscr{A}, G\left(K \otimes_{h} C_{m}\right)\right) .
\end{aligned}
$$

The second $\rightarrow$ in $(* * *)$ comes about because any $T \in \mathrm{CB}(Y, K)$ and $z \in C_{m}$ gives a map in $\mathrm{CB}\left(Y, K \otimes_{h} C_{m}\right)$ given by $y \mapsto T(y) \otimes z$. Moreover it is easy to check that this prescription gives a complete contraction ${ }_{\mathscr{B}} \mathrm{CB}(Y, K) \otimes_{h} C_{m} \rightarrow{ }_{\mathscr{B}} \mathrm{CB}\left(Y, K \otimes_{h} C_{m}\right)$. The $\cong$ in (***) comes from applying the equivalence functor.

If one checks through $(* * *)$ one finds that the composition of the maps lands up in $G\left(K \otimes_{h} C_{m}\right)$ inside $\mathscr{A} \mathrm{CB}\left(\mathscr{A}, G\left(K \otimes_{h} C_{m}\right)\right)$. That is, (***) gives a map $G(K) \otimes_{h} C_{m} \rightarrow G\left(K \otimes_{h} C_{m}\right)$. Applying $F$ to this last map and putting this together with $\left(^{*}\right)$ gives us a complete contraction

$$
K \otimes_{\min } C_{m} \cong F\left(G(K) \otimes_{h} C_{m}\right) \rightarrow F\left(G\left(K \otimes_{h} C_{m}\right)\right) \cong K \otimes_{h} C_{m}
$$

Thus we have obtained a complete contraction $K \otimes_{\min } C_{m} \rightarrow K \otimes_{h} C_{m}$ which, one can easily check, up to complete isometry, is the canonical map between these spaces. Appealing to Proposition 2.4 completes the argument of this section. 


\section{Completion of the proof of the main theorem}

Again $\mathscr{A}, \mathscr{B}, F, G, X, Y$ are as in the previous section, but now we fix $H \in$ ${ }_{\mathscr{A}} H M O D$ to be the Hilbert space of the universal representation of $\mathscr{A}$, and fix $K=F(H)$. Then $e(\mathscr{A}) \subset B(H)$, where $e(\mathscr{A})$ is the enveloping von Neumann algebra of $\mathscr{A}$. By $\S 2, F$ and $G$ restrict to an equivalence of $\mathscr{A} H M O D$ with $\mathscr{B} H M O D$. By elementary $\mathrm{C}^{*}$-algebra facts, $F$ and $G$ restricted to $H M O D$ are automatically normal *-functors in the sense of [24]. By [24] Propositions $1.1,1.3$ and 1.6, $\mathscr{B}$ acts faithfully on $K$, and if we regard $\mathscr{B}$ as a subset of $B(K)$ then the weak operator closure $\mathscr{B}^{\prime \prime}$ of $\mathscr{B}$ in $B(K)$, is $\mathrm{W}^{*}$-isomorphic to $e(\mathscr{B})$. We shall indeed regard $\mathscr{B}$ henceforth as a subalgebra of $B(K)$. We shall need the fact, from [24] Proposition 4.9, that if $H^{\infty}$ is the Hilbert space direct sum of a countably infinite number of copies of $H$, then $F\left(H^{\infty}\right) \cong K^{\infty}$, and similarly $G\left(K^{\infty}\right) \cong H^{\infty}$.

It is important in what follows to keep in mind the canonical right module action of $\mathscr{B}$ on $X . x b=F\left(r_{b}\right)(x)$, for $x \in X, b \in \mathscr{B}$, where as in the previous section $r_{b}: \mathscr{B} \rightarrow \mathscr{B}: c \mapsto c b$. Similarly, $Y$ is canonically a $\mathscr{B}-\mathscr{A}$-bimodule.

There is a left $\mathscr{B}$-module map $Y \otimes X \rightarrow F(X)$ defined by $y \otimes x \mapsto$ $F\left(r_{x}\right)(y)$. Since $F(X)=F G(\mathscr{B}) \cong \mathscr{B}$, we get a left $\mathscr{B}$-module map $Y \otimes X \rightarrow \mathscr{B}$, which we shall write as [.]. Simple algebra shows that [.] is a $\mathscr{B}-\mathscr{B}$-bimodule map, but this will not be explicitly needed. In a similar way we get a module map $(\cdot): X \otimes Y \rightarrow \mathscr{A}$. In what follows we may use the same notations for the 'unlinearized' bilinear maps, so for example we may use the symbols $[y, x]$ for $[y \otimes x]$. We now show that these maps have dense range. By way of contradiction, suppose that the closure of the range of $[\cdot]$ is a proper submodule $I$ of $\mathscr{B}$. Let $Z=\mathscr{B} / I$, regarded as a left $\mathscr{B}$-operator module (see Lemma 2.1 in [9]), and let $\pi: \mathscr{B} \rightarrow Z$ be the nonzero quotient map. Then $G(\pi): X \rightarrow G(Z)$ is nonzero. So there exists $x \in X$ such that $G(\pi) r_{x} \neq 0$. Applying $F$ we obtain $F G(\pi) F\left(r_{x}\right) \neq 0$, so that for some $y \in Y$, $F G(\pi) F\left(r_{x}\right)(y) \neq 0$. By the definition of $[\cdot]$ this implies that $\pi([y \otimes x]) \neq 0$, which contradicts the definition of $\pi$. Thus [.] (and similarly $(\cdot)$ ) has dense range.

It should be pointed out that if we are attempting to prove the main theorem, but with $O M O D$ replaced by a subcategory (as discussed in Remark 2 in $\S 1$ ), then the argument of the last paragraph seems to require that the subcategory be closed under certain quotients. However, the last paragraph can be replaced by an argument which avoids a quotient in the subcategory. Namely, pick a faithful (nonzero) representation of $\mathscr{B} / I$ on a Hilbert space $K$ say. Then $K$ can be regarded in a canonical way as an object in $\mathscr{B} H M O D$. Then there is a nonzero morphism $S$ from $\mathscr{B} / I$ to $K$. Replace the map $\pi$ in 
the previous paragraph by $S \circ \pi$, which is a nonzero morphism from $\mathscr{B}$ to $K$, and proceed in the same way.

Lemma 3.1. The canonical maps $X \rightarrow{ }_{\mathscr{B}} \mathrm{CB}(Y, \mathscr{B})$ and $Y \rightarrow \mathscr{A} \mathrm{CB}(X, \mathscr{A})$ induced by $[\cdot]$ and $(\cdot)$ respectively, are complete isometries.

Proof. Using Lemmas 2.1, 2.2, and the fact that $F(X)=F G(\mathscr{B}) \cong \mathscr{B}$, we have $X \subset \mathscr{A} \mathrm{CB}(\mathscr{A}, X) \cong{ }_{\mathscr{B}} \mathrm{CB}(Y, F(X)) \cong \mathscr{B}_{\mathrm{CB}}(Y, \mathscr{B})$ completely isometrically. Sorting through these identifications shows that an element $x \in X$ corresponds to the map $y \mapsto[y, x]$ in $\mathscr{B} C B(Y, \mathscr{B})$. A similar proof works for $(\cdot)$.

The following maps $\Phi: Y \rightarrow B(H, K)$, and $\Psi: X \rightarrow B(K, H)$ will play a central role in the remainder of the proof. Namely, $\Phi(y)(\zeta)=F\left(r_{\zeta}\right)(y)$, and $\Psi(x)(\eta)=\omega_{H} G\left(r_{\eta}\right)(x)$, where $\omega_{H}: G F(H) \rightarrow H$ is the $\mathscr{A}$-module map coming from the natural transformation $G F \cong I$. Since $\omega_{H}$ is an isometric surjection between Hilbert space it is unitary, which will be important below. It is straightforward algebra to check that:

$$
\Psi(x) \Phi(y)=(x, y) \quad \& \quad \Phi(y) \Psi(x)=[y, x] V
$$

for all $x \in X, y \in Y$, and $V \in B(K)$ is a unitary operator in $\mathscr{B}^{\prime}$ composed of two natural transformations. The $V$ will not play a significant role, since we will mostly be working with expressions such as $[y, x]^{*}[y, x]$ which by the above, and since $V$ is unitary and in $\mathscr{B}^{\prime}$, equals $\Psi(x)^{*} \Phi(y)^{*} \Phi(y) \Psi(x)$. See also [28] Lemma 4.3.

Before we begin the next lemma, we remark that for any Hilbert spaces $H, K$, since $\mathrm{CB}\left(H^{c}, K^{c}\right)=B(H, K)$ completely isometrically (see $\left.[15,6]\right)$, the norm of a matrix $\left[T_{i j}\right] \in M_{n}(B(H, K))$ can be calculated by the formula:

$$
\left\|\left[T_{i j}\right]\right\|=\sup \left\{\left\|\left[T_{i j}\left(\zeta_{k l}\right)\right]\right\|:\left[\zeta_{k l}\right] \in \operatorname{Ball}\left(M_{m}\left(H^{c}\right)\right), m \in \mathrm{N}\right\}
$$

Lemma 3.2. The map $\Phi$ (resp. $\Psi$ ) is a completely isometric $\mathscr{B}-\mathscr{A}$-module map (resp. $\mathscr{A}-\mathscr{B}$-module map). Moreover, $\Phi\left(y_{1}\right)^{*} \Phi\left(y_{2}\right) \in \mathscr{A}^{\prime \prime}=e(\mathscr{A})$ for all $y_{1}, y_{2} \in Y$, and $\Psi\left(x_{1}\right)^{*} \Psi\left(x_{2}\right) \in \mathscr{B}^{\prime \prime}$ for $x_{1}, x_{2} \in X$.

Proof. We shall simply prove the assertions for $\Phi$; those for $\Psi$ are similar. The module map assertions are fairly clear, for instance $\Phi(y a)(\zeta)=$ $F\left(r_{\zeta}\right)(y a)=F\left(r_{\zeta}\right) F\left(r_{a}\right)(y)=F\left(r_{a \zeta}\right)(y)=\Phi(y)(a \zeta)$. Next we show the $\mathscr{A}^{\prime \prime}$ assertion. By Lemma 2.2, we have a $\mathrm{C}^{*}$-isomorphism $T \mapsto F(T): \mathscr{A}^{\prime}=$ ${ }_{\mathscr{A}} \mathrm{CB}\left(H^{c}\right) \rightarrow \mathscr{B}^{\prime}={ }_{\mathscr{B}} \mathrm{CB}\left(K^{c}\right)$. Note $\Phi(y) T(\zeta)=F\left(r_{T(\zeta)}\right)(y)=F(T) F\left(r_{\zeta}\right)(y)=$ $F(T) \Phi(y)(\zeta)$, for $T \in \mathscr{A}^{\prime}$, and so also $\left(T \Phi(y)^{*}\right)^{*}=\Phi(y) T^{*}=F\left(T^{*}\right) \Phi(y)=$ $\left(\Phi(y)^{*} F(T)\right)^{*}$. Together these imply that $\Phi\left(y_{1}\right)^{*} \Phi\left(y_{2}\right) \in \mathscr{A}^{\prime \prime}$. The matching assertion for $\Psi$ has the additional complication of the maps $\omega_{H}$, however since they are unitary as remarked above, they disappear from the calcula- 
tion. Finally, we turn to the complete isometry. The equalities in the following calculation follow from, in turn, formula (2) above, the definition of $\Phi$, Lemma 2.1, Lemma 2.2, the definition of (.), formula (2) again, and Lemma 3.1:

$$
\begin{aligned}
\left\|\left[\Psi\left(y_{i j}\right)\right]\right\|= & \sup \left\{\left\|\left[\Phi\left(y_{i j}\right)\left(\zeta_{k l}\right)\right]\right\|:\left[\zeta_{k l}\right] \in \operatorname{Ball}\left(M_{m}\left(H^{c}\right)\right), m \in \mathrm{N}\right\} \\
= & \sup \left\{\left\|\left[F\left(r_{\zeta_{k l}}\right)\left(y_{i j}\right)\right]\right\|:\left[\zeta_{k l}\right] \in \operatorname{Ball}\left(M_{m}\left(H^{c}\right)\right), m \in \mathrm{N}\right\} \\
= & \sup \left\{\left\|\left[F\left(r_{\zeta_{k l}}\right) r_{y_{i j}}\right]\right\|:\left[\zeta_{k l}\right] \in \operatorname{Ball}\left(M_{m}\left(H^{c}\right)\right), m \in \mathrm{N}\right\} \\
= & \sup \left\{\left\|\left[G F\left(r_{\zeta_{k l}}\right) G\left(r_{y_{i j}}\right)\right]\right\|:\left[\zeta_{k l}\right] \in \operatorname{Ball}\left(M_{m}\left(H^{c}\right)\right), m \in \mathrm{N}\right\} \\
= & \sup \left\{\left\|\left[G F\left(r_{\zeta_{k l}}\right) G\left(r_{y_{i j}}\right)\left(x_{p q}\right)\right]\right\|:\left[\zeta_{k l}\right] \in \operatorname{Ball}\left(M_{m}\left(H^{c}\right)\right),\right. \\
& {\left.\left[x_{p q}\right] \in \operatorname{Ball}\left(M_{r}(X)\right)\right\} } \\
= & \sup \left\{\left\|\left[\left(x_{p q}, y_{i j}\right) \zeta_{k l}\right]\right\|:\left[\zeta_{k l}\right] \in \operatorname{Ball}\left(M_{m}\left(H^{c}\right)\right),\right. \\
& {\left.\left[x_{p q}\right] \in \operatorname{Ball}\left(M_{r}(X)\right), m, r \in \mathrm{N}\right\} } \\
= & \sup \left\{\left\|\left[\left(x_{p q}, y_{i j}\right)\right]\right\|:\left[x_{p q}\right] \in \operatorname{Ball}\left(M_{r}(X)\right), r \in \mathrm{N}\right\} \\
= & \left\|\left[y_{i j}\right]\right\|
\end{aligned}
$$

Thus $\Phi$ is a complete isometry.

We now proceed towards showing that

Theorem 3.3. Suppose that $\Psi(x)^{*} \Psi(x)$, which is in $\mathscr{B}^{\prime \prime}$ by the previous lem$m a$, is actually in $\mathscr{B}$ for all $x \in X$; and suppose that $\Phi(y)^{*} \Phi(y) \in \mathscr{A}$ for all $y \in Y$. Then all the conclusions of our main theorem hold.

Proof. If $\Psi(x)^{*} \Psi(x) \in \mathscr{B}$ for all $x \in X$, then by the polarization identity, and the previous lemma, $X$ is a RIGHT $C^{*}$-module over $\mathscr{B}$ with i.p. $\left\langle x_{1} \mid x_{2}\right\rangle_{\mathscr{B}}=\Psi\left(x_{1}\right)^{*} \Psi\left(x_{2}\right)$. We can also deduce that $X$ is a LEFT $\mathrm{C}^{*}$-module over $\mathscr{A}$ by setting $\mathscr{A}\left\langle x_{1} \mid x_{2}\right\rangle=\Psi\left(x_{1}\right) \Psi\left(x_{2}\right)^{*}$. This last quantity may be seen to lie in $\mathscr{A}$ by using the polarization identity and the following argument: Since the range of (.) is dense in $\mathscr{A}$, we can find a c.a.i. $\left\{e_{\alpha}\right\}$ for $\mathscr{A}$, with terms of the form $e_{\alpha}=\sum_{k=1}^{n}\left(x_{k}, y_{k}\right)=\sum_{k=1}^{n} \Psi\left(x_{k}\right) \Phi\left(y_{k}\right)$ (using equation (1)). Here $n, x_{k}, y_{k}$ depend on $\alpha$. Then $\left\{e_{\alpha}^{*}\right\}$ is also a c.a.i. for $\mathscr{A}$. Since $\Psi(x)^{*}=\lim _{\alpha} \Psi\left(e_{\alpha}^{*} x\right)^{*}=\lim _{\alpha} \Psi(x)^{*} e_{\alpha}$, it follows that $\Psi(x) \Psi(x)^{*}$ is a norm limit of finite sums of terms of the form $\Psi(x)\left(\Psi(x)^{*} \Psi\left(x_{k}\right)\right) \Phi\left(y_{k}\right)=$ $\Psi(x) \Phi\left(b y_{k}\right)=\left(x, b y_{k}\right) \in \mathscr{A}$, where $b=\Psi(x)^{*} \Psi\left(x_{k}\right) \in \mathscr{B}$. Thus $\Psi(x) \Psi(x)^{*} \in \mathscr{A}$.

A similar argument shows that $Y$ (or equivalently $\Phi(Y)$ ) is both a left and right $\mathrm{C}^{*}$-module. At this point we can therefore say that the right module actions on $Y$ and $X$ are nondegenerate. Notice also, that if we choose a contractive approximate identity for $\mathscr{A}$ of form $e_{\alpha}=\sum_{k} \Psi\left(x_{k}\right) \Phi\left(y_{k}\right)$ as 
above, then $e_{\alpha}^{*} e_{\alpha}$ is also a c.a.i. for $\mathscr{A}$. However $e_{\alpha}^{*} e_{\alpha}=\sum_{k, l} \Phi\left(y_{k}\right)^{*} b_{k l} \Phi\left(y_{l}\right)$ where $b_{k l}=\Psi\left(x_{k}\right)^{*} \Psi\left(x_{l}\right) \in \mathscr{B}$. Since $B=\left[b_{k l}\right]$ is a positive matrix, it has a square root $R=\left[r_{i j}\right]$, say, with entries $r_{i j} \in \mathscr{B}$. Thus $e_{\alpha}^{*} e_{\alpha}=\sum_{k} \Phi\left(y_{k}^{\alpha}\right)^{*} \Phi\left(y_{k}^{\alpha}\right)$, where $y_{k}^{\alpha}=\sum_{j} r_{k j} y_{j}$. From this one can easily deduce that the $\mathscr{A}$-valued innerproduct on $Y$ has dense range, that is, $Y$ is a full right $\mathrm{C}^{*}$-module over $\mathscr{A}$. Similar arguments show that $Y$ is a full left $\mathrm{C}^{*}$-module over $\mathscr{B}$, and that $X$ is also full on both sides. Thus $X$ and $Y$ are strong Morita equivalence bimodules, giving the strong Morita equivalence of $\mathscr{A}$ and $\mathscr{B}$.

Observe that by the basic theory of strong Morita equivalence (see e.g [26]) $\mathscr{A} \mathrm{K}(X) \cong \mathscr{B}$. Thus if $\left\{f_{\beta}\right\}$ is a c.a.i. for $\mathscr{B}$, then $\left\{G_{\beta}\right\}$ is a c.a.i. for ${ }_{\mathscr{A}} \mathrm{K}(X)$, where $G_{\beta}(x)=x f_{\beta}=G\left(r_{f_{\beta}}\right)(x)$. Observe too, by Lemma 2.1 , that $F(W) \cong\left\{T \in \in_{\mathscr{B}} \mathrm{CB}(B, F(W)): T r_{f_{\beta}} \rightarrow T\right.$ in norm $\}$ completely isometrically, where $\left\{f_{\beta}\right\}$ is an approximate identity for $\mathscr{B}$. Applying the functor $G$ and Lemma 2.2, we see the last set is completely isometrically isomorphic to $\left\{S \in{ }_{\mathscr{A}} \mathrm{CB}(X, G F(W)): S G\left(r_{f_{\beta}}\right) \rightarrow S\right.$ in norm $\}$, which is completely isometrically isomorphic to $\left\{S \in{ }_{\mathscr{A}} C B(X, W): S G_{\beta} \rightarrow S\right.$ in norm $\}$, which in turn equals $\mathscr{A} \mathrm{K}(X, W)$, since $G_{\beta} \in \mathscr{A} \mathrm{K}(X)$. Thus we have shown that $F(W) \cong \mathscr{A} \mathrm{K}(X, W)$ completely isometrically, and it is an easy algebra check that this is also as left $\mathscr{B}$-modules. Setting $W=\mathscr{A}$ gives $Y \cong \mathscr{A}(X, \mathscr{A})$, so that $Y \cong \bar{X}$. It is easily checked that this last relation is as bimodules too. In Theorem 3.10 in [7], we showed that $\bar{X} \otimes_{h \mathscr{A}} W \cong \mathscr{A}_{\mathfrak{A}} \mathrm{K}(X, W)$ completely isometrically. Thus $F(W) \cong Y \otimes_{h \mathscr{A}} W$ completely isometrically and as $\mathscr{B}$ modules, for all $W \in \mathscr{A} O M O D$. Its an easy algebra check now that $F \cong$ $\mathscr{A} \mathrm{K}(X,-) \cong Y \otimes_{h \mathscr{A}}-$ as functors. By symmetry, we get the matching statement for $G$. The last statement of Theorem 1.2, about the mapping of subcategories, follows because $\otimes_{h \mathscr{A}}$ coincides with the interior tensor product on the subcategories concerned.

Thus the proof of our main result has boiled down to verifying the very concrete hypotheses of the last theorem. To that end, we first observe that the natural transformations $G F(H) \cong H$ and $F G(K) \cong K$ imply certain norm equalities. Using, repeatedly, Lemmas $2.1,2.2$ and the natural transformations, we see that

$$
\begin{aligned}
H & \cong{ }_{\mathscr{A}} \mathrm{CB}(\mathscr{A}, H) \cong{ }_{\mathscr{B}} \mathrm{CB}(Y, F(H)) \cong{ }_{\mathscr{B}} \mathrm{CB}(Y, \mathscr{B} \mathrm{CB}(\mathscr{B}, F(H))) \\
& \cong \mathscr{B} \mathrm{CB}(Y, \mathscr{A} \mathrm{CB}(X, G F(H))) \cong{ }_{\mathscr{B}} \mathrm{CB}\left(Y,{ }_{\mathscr{A}} \mathrm{CB}(X, H)\right)
\end{aligned}
$$

completely isometrically. Untangling these identifications shows that $\zeta \in H$ corresponds to the following map $T_{\zeta}$ in the last space ${ }_{\mathscr{B}} \mathrm{CB}\left(Y,{ }_{\mathscr{A}} \mathrm{CB}(X, H)\right)$ in the string above: namely $T_{\zeta}(y)(x)=(x, y) \zeta$. Thus 


$$
\begin{aligned}
\|\zeta\|= & \left\|T_{\zeta}\right\|_{c b} \\
= & \sup \left\{\left\|\left[\left(x_{k l}, y_{i j}\right) \zeta\right]\right\|:\left[x_{k l}\right] \in \operatorname{Ball}\left(M_{m}(X)\right),\left[y_{i j}\right] \in \operatorname{Ball}\left(M_{n}(Y)\right), n, m \in \mathrm{N}\right\} \\
= & \sup \left\{\left\|\left[\Psi\left(x_{k l}\right) \Phi\left(y_{i j}\right) \zeta\right]\right\|:\left[x_{k l}\right] \in \operatorname{Ball}\left(M_{m}(X)\right),\right. \\
& {\left.\left[y_{i j}\right] \in \operatorname{Ball}\left(M_{n}(Y)\right), n, m \in \mathrm{N}\right\} } \\
\leq & \sup \left\{\left\|\left[\Phi\left(y_{i j}\right) \zeta\right]\right\|:\left[y_{i j}\right] \in \operatorname{Ball}\left(M_{n}(Y)\right), n \in \mathrm{N}\right\} \\
\leq & \|\zeta\|
\end{aligned}
$$

using equation (1), and the fact that $\Phi$ and $\Psi$ are complete contractions (Lemma 3.2). Thus $\|\zeta\|=\sup \left\{\left\|\left[\Phi\left(y_{i j}\right) \zeta\right]\right\|:\left[y_{i j}\right] \in \operatorname{Ball}\left(M_{n}(Y)\right), n \in \mathrm{N}\right\}$. Squaring and using the usual formula for the matrix norms on $H^{c}$ we see that

$$
\begin{aligned}
&\langle\zeta \mid \zeta\rangle=\sup \left\{\left\|\left[\Phi\left(y_{i j}\right) \zeta\right]\right\|^{2}:\left[y_{i j}\right] \in \operatorname{Ball}\left(M_{n}(Y)\right), n \in \mathrm{N}\right\} \\
&=\sup \left\{\left\|\left[\sum_{k=1}^{n}\left\langle\Phi\left(y_{k j}\right) \zeta \mid \Phi\left(y_{k i}\right) \zeta\right\rangle\right]\right\|:\left[y_{i j}\right] \in \operatorname{Ball}\left(M_{n}(Y)\right), n \in \mathrm{N}\right\} \\
&=\sup \left\{\left\|\left[\left\langle\left(\sum_{k=1}^{n} \Phi\left(y_{k i}\right)^{*} \Phi\left(y_{k j}\right)\right) \zeta \mid \zeta\right\rangle\right]\right\|:\left[y_{i j}\right] \in \operatorname{Ball}\left(M_{n}(Y)\right), n \in \mathrm{N}\right\} \\
&=\sup \left\{\left\langle\left(\sum_{k=1}^{n} \Phi\left(\sum_{i=1}^{n} y_{k i} z_{i}\right)^{*} \Phi\left(\sum_{j=1}^{n} y_{k j} z_{j}\right)\right) \zeta \mid \zeta\right\rangle:\right. \\
& {\left.\left[y_{i j}\right] \in \operatorname{Ball}\left(M_{n}(Y)\right), \sum_{i=1}^{n}\left|z_{i}\right|^{2} \leq 1\right\} }
\end{aligned}
$$

where the $z_{i} \in \mathrm{C}$. Letting $y_{k}=\sum_{i=1}^{n} y_{k i} z_{i}$ we see that

$$
\begin{gathered}
\langle\zeta \mid \zeta\rangle=\sup \left\{\left\langle\left(\sum_{k=1}^{n} \Phi\left(y_{k}\right)^{*} \Phi\left(y_{k}\right)\right) \zeta \mid \zeta\right\rangle:\right. \\
\left.\left[y_{1}, \cdots y_{n}\right]^{t} \in \operatorname{Ball}\left(C_{n}(Y)\right), n \in \mathrm{N}\right\} .
\end{gathered}
$$

Replacing $\zeta$ by $\Psi(x) \eta$ for $x \in X, \eta \in K$ we have 


$$
\begin{gathered}
\left\langle\Psi(x)^{*} \Psi(x) \eta \mid \eta\right\rangle=\sup \left\{\left\langle\left(\sum_{k=1}^{n} \Psi(x)^{*} \Phi\left(y_{k}\right)^{*} \Phi\left(y_{k}\right) \Psi(x)\right) \eta \mid \eta\right\rangle:\right. \\
\left.\left[y_{1}, \cdots y_{n}\right]^{t} \in \operatorname{Ball}\left(C_{n}(Y)\right), n \in \mathrm{N}\right\} .
\end{gathered}
$$

The expression $\sum_{k=1}^{n} \Psi(x)^{*} \Phi\left(y_{k}\right)^{*} \Phi\left(y_{k}\right) \Psi(x)$ is, by equation (1) and the remark after it, an element $b \in \mathscr{B}$, with $0 \leq b \leq \Psi(x)^{*} \Psi(x)$ since $\Phi$ is completely contractive. Thus, for $x \in X, \eta \in K$ we have

$$
\left\langle\Psi(x)^{*} \Psi(x) \eta \mid \eta\right\rangle=\sup \left\{\langle b \eta \mid \eta\rangle: b \in \mathscr{B}, 0 \leq b \leq \Psi(x)^{*} \Psi(x)\right\}
$$

A similar argument shows that for $y \in Y, \zeta \in H$, we have

$$
\left\langle\Phi(y)^{*} \Phi(y) \zeta \mid \zeta\right\rangle=\sup \left\{\langle a \zeta \mid \zeta\rangle: a \in \mathscr{A}, 0 \leq a \leq \Phi(y)^{*} \Phi(y)\right\}
$$

It follows from (5), and the fact that every quasistate of $\mathscr{A}$ has a unique $\mathrm{w}^{*}$ continuous extension to $e(\mathscr{A})$ of form $\langle\cdot \zeta \mid \zeta\rangle$ for some $\zeta \in \operatorname{Ball}(H)$, that $\Phi(y)^{*} \Phi(y)$ is a lowersemicontinuous element in $e(\mathscr{A})=\mathscr{A}^{\prime \prime}$, for each $y \in Y$. We refer the reader to [22] for details about lowersemicontinuity in the enveloping von Neumann algebra of a $\mathrm{C}^{*}$-algebra. A similar, but slightly more complicated argument, shows that $\Psi(x)^{*} \Psi(x)$, as an element in $\mathscr{B}^{\prime \prime}$, corresponds to a lowersemicontinuous element in $e(\mathscr{B})$ (which we recall, is $\mathrm{W}^{*}$-isomorphic to $\mathscr{B}^{\prime \prime}$ ). The complication occurs since it seems we can say only that the quasistates of $\mathscr{B}$ have unique $\mathrm{w}^{*}$-continuous extensions to $\mathscr{B}^{\prime \prime}$ of form $\sum_{k=1}^{\infty}\left\langle\cdot \eta_{k} \mid \eta_{k}\right\rangle$, where $\sum_{k=1}^{\infty}\left\|\eta_{k}\right\|^{2} \leq 1, \eta_{k} \in K$. Nonetheless, the calculation leading to equation (4) may be repeated, but with $H$ and $K$ replaced by $H^{\infty}$ and $K^{\infty}$ (that is, the Hilbert space direct sum of a countably infinite number of copies of $H$ or $K$ ), to yield the desired conclusion.

The crux of the proof now rests on a compactness argument in $Q(\mathscr{A})$, the (compact) set of quasistates of $\mathscr{A}$. For $y=\left[y_{1}, \cdots y_{n}\right]^{t} \in \operatorname{Ball}\left(C_{n}(Y)\right)$, and $a_{0} \in \mathscr{A}, 0 \leq a_{0} \leq 1$, set $L_{y}=a_{0}\left(\sum_{k=1}^{n} \Phi\left(y_{k}\right)^{*} \Phi\left(y_{k}\right)\right) a_{0}$, which is a lowersemicontinuous element in $e(\mathscr{A})$. Moreover, since $\Phi$ is completely contractive and since $y \in \operatorname{Ball}\left(C_{n}(Y)\right)$, we see that $L_{y} \leq a_{0}^{2}$. Replacing $\zeta$ with $a_{0} \zeta$ in (3), and using the fact that the quasistates of $\mathscr{A}$ are 'vector quasi-states' of $e(\mathscr{A})$, we see that

$$
\phi\left(a_{0}^{2}\right)=\sup \left\{L_{y}(\phi): y \in \operatorname{Ball}\left(C_{n}(Y)\right), n \in \mathrm{N}\right\}
$$

for all $\phi \in Q(\mathscr{A})$. Here $L_{y}(\phi)$ is the (scalar) value of $L_{y}$ (interpreted as an element of $\mathscr{A}^{* *}=e(\mathscr{A})$ ) evaluated at $\phi \in \mathscr{A}^{*}$. For $m \in \mathbf{N}$, and $y \in \operatorname{Ball}\left(C_{n}(Y)\right)$ set $U_{y}^{m}=\left\{\phi \in Q(\mathscr{A}): L_{y}(\phi)>\phi\left(a_{0}^{2}\right)\left(1-\frac{1}{m}\right)-\frac{1}{m}\right\}$. Since $L_{y}$ is lowersemicontinuous, $U_{y}^{m}$ is an open set in $Q(\mathscr{A})$, and by (6) for each fixed 
$m \in \mathrm{N}$, these sets $\left\{U_{y}^{m}\right\}$ form an open cover of $Q(\mathscr{A})$. Hence there is a finite subcover, corresponding to points $y_{1}^{m}, \cdots, y_{k_{m}}^{m}$.

Keeping $m$ fixed, and $x \in \operatorname{BALL}(X)$, we let $b_{k}^{m}=\Psi(x)^{*} L_{y_{k}^{m}} \Psi(x)$, which by equation (1) and the remarks after it, is an element of $\mathscr{B}$. Since each $b_{k}^{m}$ is strictly dominated (as a function on $Q(\mathscr{B})$ ) by the lowersemicontinuous function $\frac{1}{m}+\Psi(x)^{*} a_{0}^{2} \Psi(x)$, it follows by a standard lowersemicontinuity argument, effectively Dini's theorem using [22] Lemma 3.11.2, that there is an element $b^{m} \in \mathscr{B}$ satisfying $b^{m} \leq \frac{1}{m}+\Psi(x)^{*} a_{0}^{2} \Psi(x)$, and also $b^{m} \geq b_{k}^{m}-\frac{1}{m}$ for each $k$. It follows that for $\eta \in H,\|\eta\|=1$, and $x \in \operatorname{BALL}(X)$, that

$$
\begin{aligned}
\left\langle\left(\frac{1}{m}+\Psi(x)^{*} a_{0}^{2} \Psi(x)\right) \eta \mid \eta\right\rangle & \geq\left\langle b^{m} \eta \mid \eta\right\rangle \\
& \geq \max _{k}\left\langle b_{k}^{m} \eta \mid \eta\right\rangle-\frac{1}{m} \\
& =\max _{k}\left\langle\Psi(x)^{*} L_{y_{k}^{m}} \Psi(x) \eta \mid \eta\right\rangle-\frac{1}{m} \\
& =\max _{k} L_{y_{k}^{m}}\left(\phi_{0}\right)-\frac{1}{m}
\end{aligned}
$$

where $\phi_{0}(a)=\langle a \Psi(x) \eta \mid \Psi(x) \eta\rangle$. Since $x$ and $\eta$ have norm $\leq 1$, $\phi_{0}$ is a quasistate. Thus by the finite subcovering property we conclude that

$$
\begin{aligned}
\left\langle\left(\frac{1}{m}+\Psi(x)^{*} a_{0}^{2} \Psi(x)\right) \eta \mid \eta\right\rangle & \geq\left\langle b^{m} \eta \mid \eta\right\rangle \\
& \geq \phi_{0}\left(a_{0}^{2}\right)\left(1-\frac{1}{m}\right)-\frac{2}{m} \\
& =\left\langle a_{0}^{2} \Psi(x) \eta \mid \Psi(x) \eta\right\rangle\left(1-\frac{1}{m}\right)-\frac{2}{m}
\end{aligned}
$$

Thus

$$
-\frac{1}{m} \leq \Psi(x)^{*} a_{0}^{2} \Psi(x)-b^{m} \leq \frac{1}{m} \Phi(x)^{*} a_{0}^{2} \Phi(x)+\frac{2}{m} \leq \frac{3}{m}
$$

which shows that $b^{m} \rightarrow \Psi(x)^{*} a_{0}^{2} \Psi(x)$ in norm. Thus $\Psi(x)^{*} a_{0}^{2} \Psi(x) \in \mathscr{B}$. Taking $a_{0}$ to be element $e_{\alpha}$ in a c.a.i. for $\mathscr{A}$, shows that $\Psi\left(e_{\alpha} x\right)^{*} \Psi\left(e_{\alpha} x\right) \in \mathscr{B}$. Thus $\Psi(x)^{*} \Psi(x) \in \mathscr{B}$.

A similar argument (which is slightly complicated by the fact that a quasistate of $\mathscr{B}$ is of the form $\left.\sum_{k=1}^{\infty}\left\langle\cdot \eta_{k}, \eta_{k}\right\rangle\right)$, shows that $\Phi(y)^{*} \Phi(y) \in \mathscr{A}$ for $y \in Y$, which by Theorem 3.3 completes the proof.

Acknowledgements and Addenda. We particularly thank Gilles Pisier and Christian le Merdy for answering some questions related to operator 
spaces, and Vern Paulsen for various conversations, and in particular for a suggestion to move a certain trick to an earlier part of the proof (which considerably shortened the proof). Also, many of the ideas which are crucial to this paper come from our collaboration $[9,10]$ with Vern Paulsen and Paul Muhly. We thank Gert Pedersen for a discussion on lowersemicontinuity, thank others for encouragement to persist with this project, and thank the referee for his suggestions.

After finishing this paper in May 1997, we were informed that P. Ara had also obtained a characterization of strong Morita equivalence in terms of isomorphism of module categories [2,3]. However Ara works within a quite different category, namely all modules in the sense of pure algebra, both left and right sided. These modules are not over the $\mathrm{C}^{*}$-algebras, but over their Pedersen ideals. Also, the conditions on his functors (described in [3]) are also quite different. In [28] we extended our main result to possibly nonself-adjoint operator algebras. In any case, there is certainly no duplication of results or methods.

\section{REFERENCES}

1. F.W. Anderson and K.R. Fuller, Rings and Categories of Modules (2nd Ed.), Graduate Texts in Math. 13, 1992.

2. P. Ara, Morita equivalence and Pedersen's ideals, Proc. Amer. Math. Soc. (To appear).

3. P. Ara, Morita equivalence for rings with involution, Algebras Representation Theory 2 (1999), 227-247.

4. H. Bass, The Morita Theorems, Lecture Notes, University of Oregon, Eugene, 1962.

5. W. Beer, On Morita equivalence of nuclear $C^{*}$-algebras, J. Pure Appl. Algebra 26 (1982), 249-267.

6. D. P. Blecher, Tensor products of operator spaces II, Canad. J. Math. 44 (1992), 75-90.

7. D. P. Blecher, A generalization of Hilbert modules, J. Funct. Anal., 136 (1996), 365-421.

8. D. P. Blecher, A new approach to Hilbert $C^{*}$-modules, Math Ann. 307 (1997), 253-290.

9. D. P. Blecher, P. S. Muhly and V. I. Paulsen, Categories of operator modules - Morita equivalence and projective modules, Mem. Amer. Math. Soc. Vol. 143 number 681 January 2000.

10. D. P. Blecher, P. S. Muhly and V. I. Paulsen, Morita equivalence for operator algebras II, In preparation.

11. D. P. Blecher and V. Paulsen, Tensor products of operator spaces, J. Funct. Anal. 99 (1991), 262-292.

12. A. Connes, Noncommutative Geometry, Academic Press (1994).

13. E. Christensen, E. Effros, and A. Sinclair, Completely bounded multilinear maps and $C^{*}$-algebraic cohomology, Invent. Math. 90 (1987), 279-296.

14. E. Effros and Z. J. Ruan, Representations of operator bimodules and their applications, J. Operator Theory 19 (1988), 137-157.

15. E. Effros and Z. J. Ruan, Self-duality for the Haagerup tensor product and Hilbert space factorization, J. Funct. Anal. 100 (1991), 257-284.

16. E. Effros and Z. J. Ruan, The Grothendieck-Pietsch and Dvoretzky-Rogers Theorem for Operator Spaces, J. Funct. Anal. 122 (1994), 428-450. 
17. N. Gronbaek, Morita equivalence for Banach algebras, a transcription, J. Pure Appl. Algebra 99 (1995), 183-219.

18. E. C. Lance, Hilbert $C^{*}-$ modules $-A$ toolkit for operator algebraists, London Math. Soc. Lecture Note Ser., 1995.

19. W. Paschke, Inner product modules over $B^{*}$-algebras, Trans. Amer. Math. Soc. 182 (1973), 443-468.

20. V. Paulsen, Completely bounded maps and dilations, Pitman Res. Notes in Math.,1986.

21. V. I. Paulsen and R. R. Smith, Multilinear maps and tensor norms on operator systems, J. Funct. Anal. 73 (1987), 258-276.

22. G. Pedersen, $C^{*}$-algebras and their Automorphism Groups, Academic Press (1979).

23. G. Pisier, The operator Hilbert space OH, complex interpolation and tensor norms, Mem Amer. Math. Soc. 585 (1996).

24. M. Rieffel, Morita equivalence for $C^{*}$-algebras and $W^{*}$-algebras, J. Pure Appl. Algebra 5 (1974), 51-96.

25. M. Rieffel, Induced representations of $C^{*}$-algebras, Adv. Math. 13 (1974), 176-257.

26. M. Rieffel, Morita equivalence for operator algebras, Proc. Sympos. Pure Math. 38 Part 1 (1982), 285-298.

27. G. Wittstock, Extensions of completely bounded module morphisms, Proceedings of conference on operator algebras and group representations, Neptum, Pitman (1983).

28. D. P. Blecher, A Morita theorem for algebras of operators on Hilbert Space, J. Pure Appl. Algebra (to appear).

\author{
DEPARTMENT OF MATHEMATICS \\ UNIVERSITY OF HOUSTON \\ HOUSTON TX 77204-3476 \\ USA \\ Email: dblecher@math.uh.edu
}

